\title{
Role of HSP90 $\alpha$ in osteoclast formation and osteoporosis development
}

\author{
JIANLI MA ${ }^{1^{*}}$, CHEN YANG $^{2^{*}}$, HUAJIAN ZHONG ${ }^{2 *}$, CHENG WANG $^{3}$, \\ $\mathrm{KE} \mathrm{ZHANG}^{2}$, XIAOMING $\mathrm{LI}^{4}$, JINHUI WU ${ }^{2}$ and YANG GAO ${ }^{3}$ \\ ${ }^{1}$ Department of Pharmacy, Chinese PLA General Hospital, Medical School of Chinese PLA, Beijing 100039; \\ ${ }^{2}$ Department of Orthopedics, Changzheng Hospital, Shanghai 200003; ${ }^{3}$ Department of Orthopedics, \\ National Clinical Research Center for Orthopedics, Sports Medicine and Rehabilitation, \\ Chinese PLA General Hospital, Medical School of Chinese PLA, Beijing 100039; \\ ${ }^{4}$ Department of Orthopedics, Shanghai Hospital, Shanghai 200433, P.R. China
}

Received September 2, 2020; Accepted April 20, 2021

DOI: $10.3892 /$ etm.2022.11199

\begin{abstract}
Osteoporosis (OP) is a systemic metabolic bone disease that occurs most frequently in the elderly. The main pathogenesis of OP is excessive proliferation and differentiation of osteoclasts, in which the peroxisome proliferator-activated receptor $\gamma(\operatorname{PPAR} \gamma)$ pathway has a pivotal role. Recently, heat shock protein (HSP) $90 \alpha$ has been identified as an important molecular chaperone with PPAR $\gamma$, which regulates the effect of the PPAR $\gamma$ pathway. The aim of the present study was to investigate the role of HSP90 $\alpha$ involved in the regulation of osteoclast formation and the process of osteoporosis. Firstly, the expression of HSP90 $\alpha$ in osteoclast differentiation was detected by western blotting in vitro, then the effect of HSP90 $\alpha$ inhibition on the formation and differentiation of osteoclasts was examined. Furthermore, the nuclear import of PPAR $\gamma$ was also assessed to confirm the synergistic effect of HSP90 $\alpha$. Finally, the inhibitory effect of HSP90 $\alpha$ in vivo was explored, using a mouse model of osteoporosis. As a result, in the process of osteoclast differentiation and proliferation, the expression of HSP90 $\alpha$ was upregulated. Inhibition of HSP90 $\alpha$ could block the formation and differentiation of
\end{abstract}

Correspondence to: Professor Jinhui Wu, Department of Orthopedics, Changzheng Hospital, 415 Fengyang Road, Shanghai 200003, P.R. China

E-mail: a1214123123@sina.com

Professor Yang Gao, Department of Orthopedics, National Clinical Research Center for Orthopedics, Sports Medicine and Rehabilitation, Chinese PLA General Hospital, Medical School of Chinese PLA, 28 Fuxing Road, Beijing 100039, P.R. China

E-mail: gyang_2018@163.com

*Contributed equally

Key words: osteoporosis, osteoclast, nuclear import of peroxisome proliferator-activated receptor $\gamma$, heat shock protein 90 osteoclasts, and remit osteoporosis in mice. Regarding the underlying mechanism, inhibition of HSP90 $\alpha$ could block the nuclear import of PPAR $\gamma$ to inhibit osteoclast differentiation and proliferation. In conclusion, these data indicated that the inhibition of HSP90 $\alpha$ could block osteoclast formation and remit osteoporosis by reducing the nuclear import of PPAR $\gamma$.

\section{Introduction}

Osteoporosis (OP) is a systemic metabolic bone disease characterized by loss of bone mass, bone microstructural damage, decreased bone strength, and increased risk of bone fractures. It occurs more frequently in the elderly for whom it is one of the main causes of disability. The aging of society, living environment, dietary habits, as well as other factors contribute to the increased incidence of $\mathrm{OP}(1,2)$. The main pathogenesis of OP is the proliferation and differentiation of osteoclasts. The formation and inhibition of osteoclasts are associated with multiple signaling pathways, including the OPG/RANKL/RANK signaling pathway, tumor necrosis factor signaling pathway, nitric oxide and estrogen, peroxisome proliferator-activated receptor $\gamma(\operatorname{PPAR} \gamma)$, and microphthalmia-associated transcription factor $(3,4)$. Among these pathways, the PPAR $\gamma$ pathway has a pivotal role in osteoclast formation and the mechanism of OP caused by its excessive activation. Previous studies have demonstrated that activated PPAR $\gamma$ could promote osteoclast differentiation and inhibit the differentiation of mesenchymal stem cells into osteoblasts $(5,6)$. Furthermore, animal experiments demonstrated that knockout of the PPAR $\gamma$ gene could lead to severe osteosclerosis in mice, which indicates that differentiation and activity of osteoclasts were significantly inhibited (7). Thus, the application of PPAR $\gamma$ as a target for inhibiting osteoclast formation could be an important strategy for OP treatment.

Over recent years, previous studies have found that the ligand of PPAR $\gamma$ must first bind to heat shock protein (HSP) $90 \alpha$ and then polymerize with PPAR $\gamma(8,9)$. It has been suggested that HSP90 $\alpha$ may be an important molecule affecting PPAR $\gamma$. Therefore, it was theorized that HSP90 $\alpha$ may affect the 
formation and activation of osteoclasts by regulating PPAR $\gamma$, and participating in the occurrence of OP. Thus, the aim of this study was to investigate the relationship between HSP90 $\alpha$ and osteoclasts, and explore their association and the effect on OP in mice through in vivo and in vitro experiments.

\section{Materials and methods}

THP-1 human monocytic cell line was purchased from American Type Culture Collection (cat. no. TIB-202). Mouse anti-PPAR $\gamma$ antibody (product code ab41928), rabbit anti-tartrate-resistant acid phosphatase (TRAP) antibody (product code ab65854) and rabbit anti-HSP90 $\alpha$ antibody (product code ab79849) were acquired from Abcam. Rabbit anti-GAPDH (cat. no. ab8245; Abcam) antibody, HRP-labeled goat anti-rabbit IgG antibody (cat. no. \#7074S) and goat anti-mouse IgG antibody (cat. no. \#7076S)were provided by Cell Signaling Technology, Inc. Alvespimycin was obtained from MedChemExpress (product no. HY-10389). High-glucose Dulbecco's modified Eagle medium (Dulbecco's modified Eagle medium, DMEM), fetal bovine serum (FBS) and other reagents for cell culture were provided by Gibco; Thermo Fisher Scientific, Inc. TRAP kits were products of Sigma-Aldrich; Merck KGaA (cat. no. 387A). Cell lysis buffer, protease inhibitors and other reagents were purchased from Beyotime Institute of Biotechnology. Chemiluminescence detection reagent pro-light HRP was purchased from MilliporeSigma.

THP-1 cell-induced differentiation. THP-1 cells were seeded in a 24 -well plate $\left(1 \times 10^{6}\right.$ cells/well). After cell attachment $(\sim 4 \mathrm{~h})$, cells were cultured with normal RPMI-1640 medium and a humidified atmosphere containing $5 \% \mathrm{CO}_{2}$ at $37^{\circ} \mathrm{C}$. The next day, cells were treated with 1,000 units of M-CSF, PMA $(5 \mathrm{ng} / \mathrm{ml}$ ) and $50 \mathrm{ng} / \mathrm{ml}$ soluble receptor activator of $\mathrm{NF}-\kappa \mathrm{B}$ ligand (sRANKL) was added to the culture medium. After 4 days, it was observed if the cells had coenocytes and TRAP staining was performed after 5 days of induction.

Anti-TRAP staining. The old culture medium in the 24-well plate was discarded. Cells $\left(1 \times 10^{5}\right)$ were then gently washed two times with sterile ice PBS, fixed with paraformaldehyde (4\%) at room temperature for $30 \mathrm{sec}$ and was stained with hematoxylin for $1 \mathrm{~h}$ in a water bath at $37^{\circ} \mathrm{C}$. Glycerol was used for mounting, and an inverted light microscope was used for observation.

Cell counting kit-8 (CCK-8) assay. The treated THP-1 cells (5,000/well) in RPMI-1640 medium containing $10 \%$ FBS were seeded into a 96 -well plate (100 $\mu \mathrm{l} /$ well). Each well was then mixed with $10 \mu \mathrm{l}$ of CCK-8 (cat. no. CK04; Dojindo Molecular Technologies, Inc.) at $37^{\circ} \mathrm{C}$ and $5 \% \mathrm{CO}_{2}$ in the dark for $1 \mathrm{~h}$. The OD value was measured at $450 \mathrm{~nm}$ on a microplate reader at 0 - and 1-h time-points.

Detection of proliferation by CFDA SE-labeling and flow cytometry analysis. After the cells were treated with alvespimycin $(20 \mathrm{nM})$ for $24 \mathrm{~h}$, they were collected by and washed once with PBS; and then with a $1 \mathrm{X}$ Buffer A kit (cat. no. C1031; Beyotime Institute of Biotechnology) according to the manufacturer's instructions. After collection, the cell concentration was adjusted to $1 \times 10^{6}$ cells $/ \mathrm{ml}$. The cells were collected by centrifugation ( $447 \mathrm{x} \mathrm{g} ; 3 \mathrm{~min} ; 25^{\circ} \mathrm{C}$ ), washed with $1 \mathrm{X}$ Buffer A and resuspended in $500 \mu 1 \mathrm{1X}$ Buffer A. Then, $5 \mu \mathrm{l}$ CFDA-SE fluorescence probe (cat. no. C1031; Beyotime Institute of Biotechnology) was added for staining for $30 \mathrm{~min}$ in the dark at room temperature. Cells were then transferred to the flow cytometer tube and detection on the instrument was performed. CFDA SE was excited by a 488-nm argon-ion laser and analyzed using a 630-nm band-pass filter. Finally, cells were collected through the FSC/SSC scatter plot. Gating technology was used to exclude adherent cells and fragments. Finally, Flowjo software (version 10.6.0; FlowJo LLC) was used to obtain the proportion of cells in second generation, and then a histogram was constructed.

Western blot analysis. The total protein of cells and tissues was extracted with RIPA cell lysis buffer (Beyotime Institute of Biotechnology) and the protein concentration was determined by the BCA method. The protein loading amount at $40 \mathrm{mg} /$ lane was subjected to SDS-PAGE (10\%). The protein was transferred to a PVDF membrane by wet transfer at a constant voltage of $70 \mathrm{~V}$ for $1 \mathrm{~h}$. Next, $5 \%$ skim milk powder was used for blocking at room temperature for $2 \mathrm{~h}$. Samples were then incubated with the primary antibodies (anti-HSP90 $\alpha, 1: 1,000$; and anti-PPAR $\gamma, 1: 500)$ at $4^{\circ} \mathrm{C}$ overnight and then with the secondary antibodies $(1: 3,000)$ at room temperature for $2 \mathrm{~h}$. ECL chemiluminescence was used to detect protein expression. Densitometry was quantified by ImageJ software (v1.8.0; National Institutes of Health).

Establishment of a mouse model of OP after ovariectomy. A total $25 \mathrm{Balb} / \mathrm{c}$ male mice, 6-8 weeks old, weighing 20-25 g, were obtained from Lingchang Biotechnology Co., Ltd. All the animals were housed in an environment with a temperature of $22 \pm 1^{\circ} \mathrm{C}$, relative humidity of $50 \pm 1 \%$, and a 12 -h light/dark cycle. All the animals had free access to food and water. All animal studies (including the procedure for euthanasia of mice) were performed in compliance with the regulations and guidelines of the Laboratory Animal Center and Ethics Committee of Changzheng Hospital (approval ID, \#CZ2019112130) and conducted according to the AAALAC and the IACUC guidelines.

Mice weight was monitored for more than 3 days. Ovariectomy was performed after no significant change in weight was detected. Before surgery, surgical instruments were disinfected with a high temperature, including tissue scissors, tissue forceps, tweezers, needle holders, cotton balls and rubber bands; disposable surgical blades, sutures and surgical towels were obtained from the Department of Instruments of Changzheng Hospital.

The laboratory mice were anesthetized by intraperitoneal injection of $3 \%$ pentobarbital sodium solution $(50 \mathrm{mg} / \mathrm{kg}$ weight). After anesthesia was completed, the mice were placed in the prone position on the operating table, and their four limbs and front teeth were fixed. A surgical blade was used to prepare the skin in the lower back, after which the lower back was disinfected with iodophor, and a surgical towel was draped. Scissors were used to produce a longitudinal incision of $\sim 1 \mathrm{~cm}$ in the back of mice away from the lumbar spine. The lower back muscles were cut on both sides, and the ovaries 
were exposed. The mouse ovaries were red cauliflower-shaped, surrounded by adipose tissues, containing fallopian tubes and blood vessels. For sham-operated mice, the ovaries were removed and immediately placed back to the abdominal cavity. For ovariectomized mice, the fallopian tubes and blood vessels were ligated, and the ovaries and surrounding adipose tissues were removed. After confirming that the ovaries were completely removed, the stump of the fallopian tube was disinfected and placed back to the abdominal cavity. The muscles were sutured layer by layer. Disinfection was performed again to suture the skin. Picric acid was applied to the skin wound, and the numbered ear studs were fixed on the left ear of the mice for labeling. The mice were then placed into cages. After the mice were fully awakened, they were transferred to the breeding place, and the feeding was continued.

On day 42 after the ovaries were removed, mice were euthanized by $70 \%$ carbon dioxide inhalation, and death was confirmed by verifying respiratory and cardiac arrest. Then their bone tissues were fixed in $4 \%$ polyformaldehyde at room temperature for $48 \mathrm{~h}$, and decalcified in 10\% EDTA at room temperature for 10-14 days. The decalcified bone tissues were embedded in paraffin and were sectioned with mean border thickness of $1.9 \mathrm{~mm}$ and then dehydrated in $70 \%$ ethanol for $30 \mathrm{~min}, 80 \%$ ethanol for $30 \mathrm{~min}, 90 \%$ ethanol three times for $1 \mathrm{~h}$ and $100 \%$ ethanol twice for $2 \mathrm{~h}$ at room temperature, cleared in xylene and paraffin embedded. Cross-sections $(6 \mu \mathrm{m})$ were prepared for staining with H\&E and Masson's trichrome at room temperature for histological evaluation that was observed by light microscope (Eclipse 80i; Nikon Corporation) and was analyzed by the ImageJ software (v1.8.0; National Institutes of Health).

$H \& E$ staining. Cross-sections $(6 \mu \mathrm{m})$ of paraffin-embedded bone tissues were prepared. Firstly, xylene was used to remove paraffin from the sections, and subsequently the sections were rehydrated into ethanol (from 90-70\%). After being washed with water, the sections were placed in hematoxylin aqueous solution for a few min. Subsequently, the sections were incubated in acid water and ammonia water for a few secs, washed with water for $1 \mathrm{~h}$, placed into ethanol (from 70-90\%) for $10 \mathrm{~min}$, and finally incubated into alcohol eosin staining solution for 2-3 min. After transparency with xylene, the sections were mounted with Canadian gum, covered with a cover glass and sealed, and observed under a light microscope. All the procedures were performed at room temperature.

Masson's trichrome staining. Bone paraffin sections were routinely deparaffinized to water as aforementioned and stained with Weigert's iron hematoxylin staining solution for 5-10 $\mathrm{min}$, followed by acid ethanol differentiation solution for 5-15 sec and washing with water. Subsequently, the sections were stained with Masson's blue solution for 3-5 min and washed with water. Then, the sections were washed with distilled water for $1 \mathrm{~min}$, and stained with Ponceau red magenta staining solution for 5-10 min. The sections were washed with a weak acid solution (ratio of distilled water: Weak acid solution=2:1) for $1 \mathrm{~min}$, and subsequently washed with phosphomolybdic acid solution for 1-2 min, followed by washing with the prepared weak acid working solution for $1 \mathrm{~min}$, and directly placed into aniline blue staining solution for 1-2 min, washed with the prepared weak acid working solution for $1 \mathrm{~min}$, quickly dehydrated with $95 \%$ ethanol and three times with anhydrous ethanol (5-10 sec each), followed by three times incubation in xylene (1-2 min each), and finally sealed with neutral gum and observed under a light microscope. All the procedures were performed at room temperature.

Immunofluorescence assay. The THP-1 cells were seeded in 24-well plates $\left(2 \times 10^{5} /\right.$ well), washed twice with PBS, fixed with cold acetone/methanol (1:1) for $20 \mathrm{~min}$ and blocked at room temperature with $10 \%$ normal goat serum for $1 \mathrm{~h}$. The anti-HSP90A $\alpha(1: 1,000)$ and anti-PPAR $\gamma(1: 1,000)$ antibodies were separately added, and incubated at $4^{\circ} \mathrm{C}$ in a wet box overnight. A FITC-labeled goat anti-mouse secondary antibody (1:3,000; cat. no. AP130F; Sigma-Aldrich; Merck KGaA) was added dropwise and incubated for $1 \mathrm{~h}$ at room temperature in the dark. A total of $20 \%$ glycerol was added for mounting, and images were captured and observed on a confocal microscope (Leica Microsystems GmbH). PBS was used as a negative control. Green and red fluorescence were used as a positive expression; DAPI was added and stained the nucleus at room temperature for $30 \mathrm{~min}$.

HSP90 $\alpha$ inhibitor application in vivo and in vitro. Alvespimycin is a common inhibitor of HSP90 $\alpha$ that has been reported in numerous studies (10-12), and it was used in further experiments in vivo and in vitro. Briefly, for the mouse model of OP, alvespimycin was injected intraperitoneally with a dosage of $25 \mathrm{mg} / \mathrm{kg}$ five times a week according to a study by Mellatyar et al (11). For experiments in vitro, alvespimycin at $20 \mathrm{nM}$ was used to inhibit $\operatorname{HSP} 90 \alpha(13,14)$.

Statistical analysis. All experiments were repeated in triplicate to ensure the reliability of the results. All data were presented as the mean \pm SD. SPSS 19.0 (IBM Corp.) software package was used for statistical analysis. Comparisons between two groups were performed using unpaired t-tests. Multiple groups were analyzed using one-way analysis of variance and post hoc unpaired Tukey's tests. $\mathrm{P}<0.05$ was considered to indicate a statistically significant difference.

\section{Results}

Expression of HSP90 $\alpha$ in osteoclast differentiation. To explore the role of HSP90 $\alpha$ in osteoclast differentiation, western blotting was used to detect the expression. THP-1 monocytes, which were precursors of osteoclasts, were cultured for 5 days under the induction of PMA, M-CSF, and RANKL. All THP-1 monocytes differentiated into TRAP $(+)$ multinuclear osteoclasts (Fig. 1A and B). Further analysis confirmed that in the RANKL-induced osteoclast differentiation, the expression level of HSP $90 \alpha$ reached a peak at $72 \mathrm{~h}$, and then gradually decreased. At $120 \mathrm{~h}$, the expression level of HSP90 $\alpha$ was decreased compared with the 72-h treatment. Concurrently, the expression level of PPAR $\gamma$ was increased, and that of HSP90 $\alpha$ was also upregulated. (Fig. 1C-E).

Inhibition ofHSP90 $\alpha$ canblock the formation and differentiation of osteoclasts. To further confirm the effect of HSP90 $\alpha$ on 

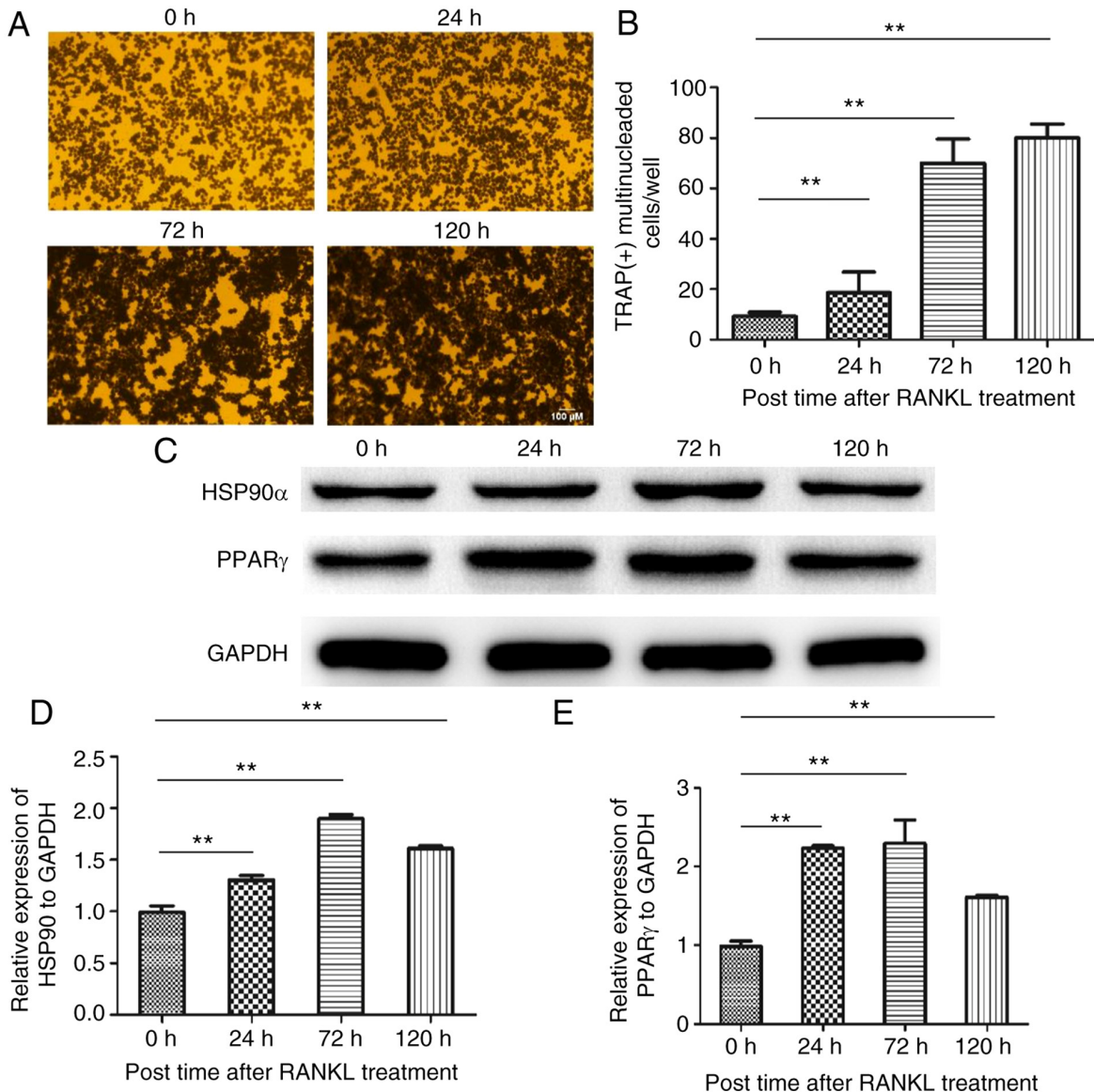

Post time after RANKL treatment
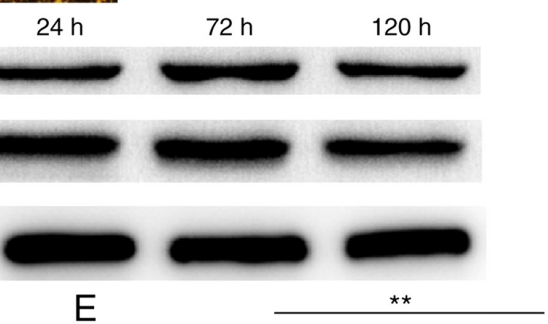

$\mathrm{E}$

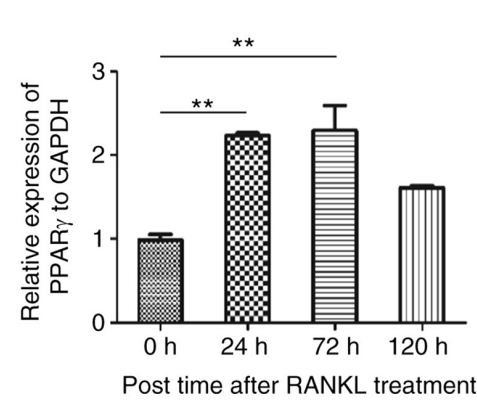

Figure 1. Expression of HSP90 $\alpha$ in the osteoclast differentiation. THP-1 cells were treated with M-CSF (1,000 units), PMA ( $5 \mathrm{ng} / \mathrm{ml})$ and sRANKL (50 ng/ml). (A and B) At different time-points ( $0,24,72$ and $120 \mathrm{~h}$ ), THP-1 cells were stained by anti-TRAP to identify osteoclast differentiation. (C-E) Concurrently, THP-1 cells were collected to detect the expression of HSP90 $\alpha$ and PPAR $\gamma$ by western blotting. Data are expressed as the mean \pm SD of three separate experiments. ${ }^{* *} \mathrm{P}<0.01$. HSP, heat shock protein; PPAR $\gamma$, peroxisome proliferator-activated receptor $\gamma$.

osteoclast formation and differentiation, alvespimycin was used to inhibit HSP90 $\alpha$. The results revealed that alvespimycin had no significant effect on the number of TRAP $(+)$ multinuclear osteoclasts in the uninduced culture. When induced, alvespimycin significantly reduced the number of TRAP $(+)$ multinuclear osteoclasts, indicating that inhibition of HSP90 $\alpha$ may inhibit the activation of osteoclasts (Fig. 2A and B). In order to further verify the aforementioned results, the expression levels of TRAP and PPAR $\gamma$ in THP-1 monocytes were detected by western blotting. The results were consistent with those of TRAP staining. Alvespimycin significantly reduced the expression level of TRAP after induction, but had no significant effect on the expression of PPAR $\gamma$ (Fig. 2C-E). The aforementioned findings indicated that inhibition of HSP90 $\alpha$ could block the formation and differentiation of osteoclasts, but had no significant effect on the expression of PPAR $\gamma$.

Inhibition of HSP90 $\alpha$ can exert an inhibitory effect on osteoclasts by blocking nuclear import of PPAR $\gamma$. Previous experiments revealed that inhibition of HSP90 $\alpha$ significantly inhibited osteoclast differentiation, but had no effect on PPAR $\gamma$ expression. It has been indicated that PPAR $\gamma$ enters the nucleus and initiates the transcription of the corresponding genes (15). Therefore, it was theorized in the present study that HSP90 $\alpha$ may regulate the differentiation of osteoclasts by affecting the nuclear import of PPAR $\gamma$. Thus, a confocal microscope was used to observe the nuclear import of PPAR $\gamma$ (red) and HSP90 $\alpha$ (green) after RANKL induction. The results demonstrated that after induction by PMA, M-CSF, and RANKL for $1 \mathrm{~h}$, nuclear import of HSP90 $\alpha$ (red) and PPAR $\gamma$ (green) in THP-1 monocytes was markedly increased. However, after inhibition of HSP90 $\alpha$ by alvespimycin, the nuclear import was markedly decreased (Fig. 3). These data indicated that the inhibition of HSP90 $\alpha$ exerted an inhibitory effect on osteoclasts by blocking the nuclear import of PPAR $\gamma$.

Effect of HSP90 $\alpha$ on the biological proliferative ability of osteoclasts. The aforementioned experiments demonstrated that inhibition of HSP $90 \alpha$ could inhibit osteoclast differentiation, and the effect of inhibition of HSP90 $\alpha$ on the proliferation of osteoclast precursor monocytes was further observed. THP-1 monocytes were treated with alvespimycin. Results of the CCK- 8 assay revealed that THP-1 monocytes had a significant decrease in viability $12 \mathrm{~h}$ after alvespimycin treatment, and continued to decrease over time (Fig. 4A). Furthermore, flow cytometric results revealed that after $24 \mathrm{~h}$ of alvespimycin treatment, the proportion of second-generation for CFDA SE-labeled cells significantly decreased $(92.51 \pm 1.39$ vs. $83.55 \pm 2.30)$, indicating that the proliferation ability was reduced (Fig. 4B and C). The aforementioned results indicated that inhibition of HSP90 $\alpha$ could block the proliferation of osteoclast precursor monocytes. 

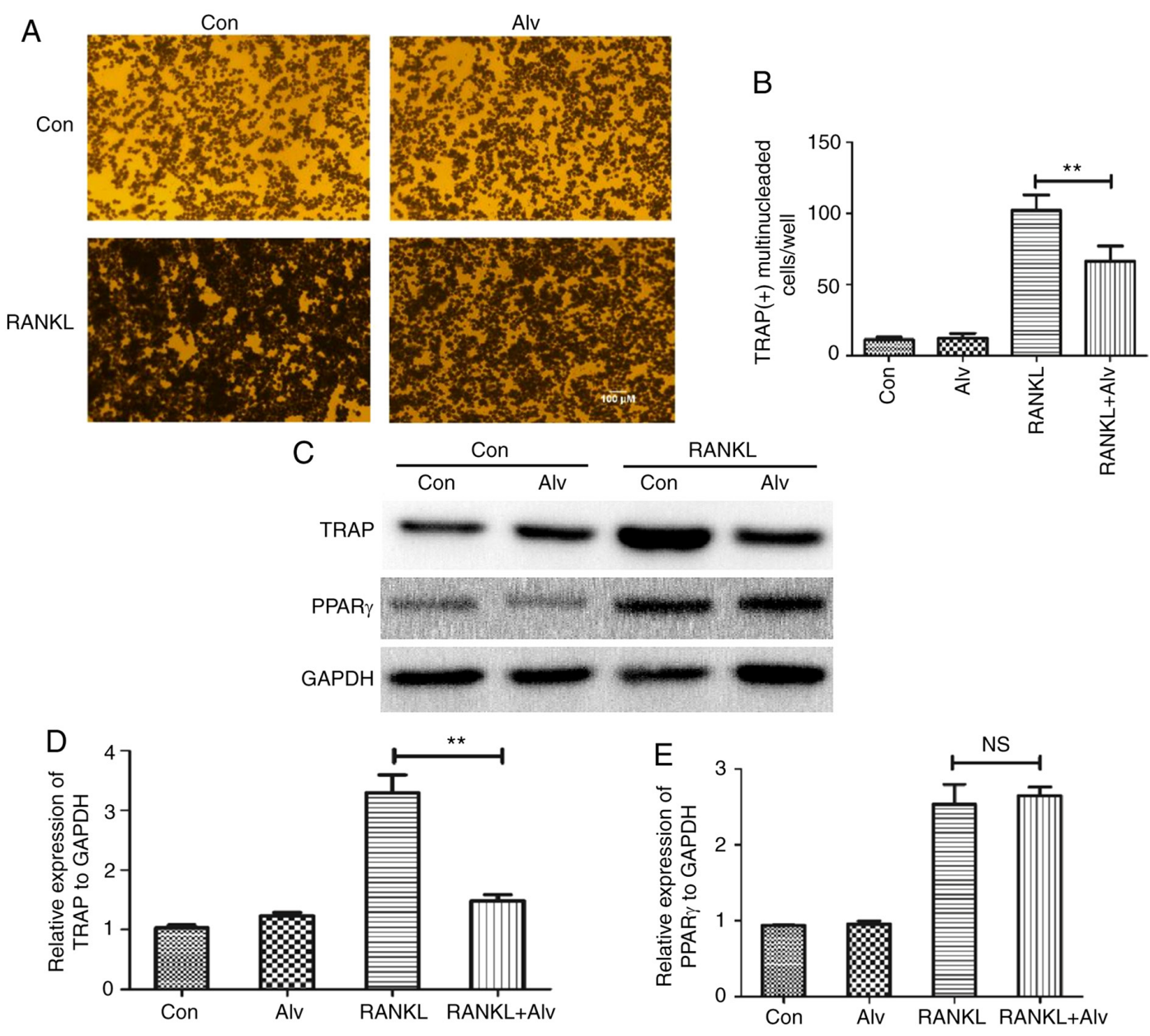

Figure 2. Inhibition of HSP $90 \alpha$ can block the formation and differentiation of osteoclasts. THP-1 monocytes were induced by sRANKL ( $50 \mathrm{ng} / \mathrm{ml}) \mathrm{with} / \mathrm{without}$ alvespimycin $(20 \mathrm{nM})$ for 5 days. (A and B) Then, the cells were stained by anti-TRAP to identify osteoclast differentiation. (C-E) Concurrently, THP-1 cells were collected to detect the expression of TRAP and PPAR $\gamma$ by western blotting. Data are expressed as the mean \pm SD of three separate experiments. ${ }^{* *} \mathrm{P}<0.01$. HSP, heat shock protein; PPAR $\gamma$, peroxisome proliferator-activated receptor $\gamma$; NS, not significant; Con, control; Alv, alvespimycin.

Inhibition of HSP90 $\alpha$ can improve OP in mice. The aforementioned experiments demonstrated that inhibition of HSP90 $\alpha$ could inhibit the differentiation and proliferation of osteoclasts, which are the bases of OP. Therefore, the effect of inhibition of HSP $90 \alpha$ on OP in mice was further studied. A mouse model of OP was constructed by removing mouse ovaries. The results revealed that on day 42 after the ovaries were removed, the bone mass of mice decreased, the bone cortex became thinner, the trabecular bone was decreased, deformed and thinned, and arrangement was disordered. In addition, the bones were porous and deficient in calcium, which suggested the presence of OP. After treatment with alvespimycin $(25 \mathrm{mg} / \mathrm{kg})$, the bone mass became thicker and the trabeculae was increased compared with the sham-operated group. Moreover, a lower TRAP(+) rate in mice was found in the alvespimycin treatment group (Fig. 5A and B), indicating less osteoclast differentiation. Concurrently, the results revealed that the red area of Masson staining of the bone in the model group was decreased, indicating the reduced bone maturity. After treatment with alvespimycin, the red area increased, suggesting that inhibition of HSP90 $\alpha$ by alvespimycin can inhibit osteoclast differentiation, promote bone maturation, thus improving OP (Fig. 5C and D).

\section{Discussion}

Heat shock proteins (HSPs) are potent antioxidative and anti-inflammatory proteins that protect cells from numerous acute and chronic stress conditions; HSPs can act as molecular chaperones that prevent the aggregation of misfolded proteins, rendering these proteins refolded or removed (16). Studies have revealed that PPAR $\gamma$ can exert its biological role by regulating a variety of HSPs (such as HSP90, HSP70, HSP60, and HSP32) (17). Nguyen et al (18) found that the function of HSP90 $\alpha$ was essential for PPAR $\gamma$ transcription output and survival of mature adipose cells. Moreover, it was revealed that HSP90 $\alpha$ can bind to PPAR $\gamma$ to form an HSP-PPAR $\gamma$ compound, controlling the stability of PPAR $\gamma$ and cell differentiation (18). Lee et al (19) discovered that binding of ox-LDL to the receptor CD36 could inhibit HSP expression at the translation level through PPAR $\gamma$ activation. Moreover, Chen et al (20) revealed that nifedipine could inhibit the phosphorylation of p38MAPK, ERK1/2 and HSP90 by promoting the expression of PPAR $\beta / \gamma$, thereby inhibiting the production of MMP-2 and CD40L and exerting its anti-AS effect. The aforementioned studies demonstrated that HSP90 $\alpha$ has a pivotal role in the regulation of PPAR $\gamma$ function, and that PPAR $\gamma$ is an important driving factor for osteoclast formation 

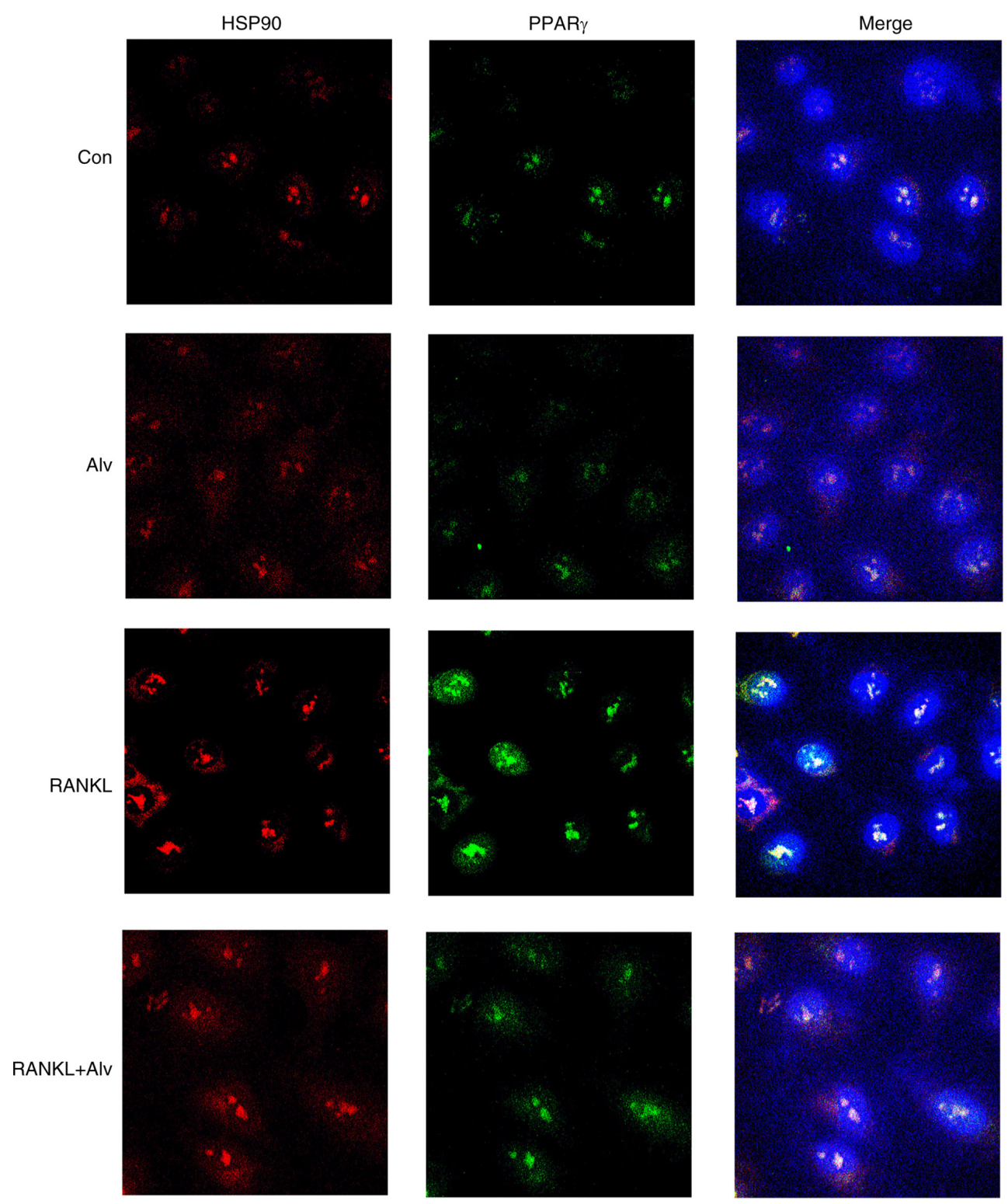

Figure 3. Inhibition of HSP90 $\alpha$ can exert an inhibitory effect on osteoclasts by blocking nuclear entry of PPAR $\gamma$. THP-1 monocytes were induced by sRANKL $(50 \mathrm{ng} / \mathrm{ml})$ with/without alvespimycin $(20 \mathrm{nM})$ for $1 \mathrm{~h}$, then the nuclear (blue) import of PPAR $\gamma$ (red) and location of HSP90 $\alpha$ (green) were observed with confocal immunofluorescence. HSP, heat shock protein; PPAR $\gamma$, peroxisome proliferator-activated receptor $\gamma$; Con, control; Alv, alvespimycin.

and differentiation. Therefore, in the present study, it was further examined whether HSP90 $\alpha$ could affect the function of osteoclasts, and further regulate the formation of OP.

Firstly, the expression level of HSP90 $\alpha$ during osteoclast formation and differentiation in vitro was examined, and it was determined that after induction by PMA, M-CSF, and RANKL, the number of TRAP(+) multinuclear osteoclasts differentiated by THP-1 monocytes was gradually increased. Concurrently, the expression level of HSP90 $\alpha$ was also gradually increased. These results indicated that there may be an association between the two. On this basis, HSP90 $\alpha$ inhibitor alvespimycin was used to intervene on THP-1 monocytes, and the results revealed that alvespimycin could significantly inhibit the differentiation of THP-1 monocytes into osteoclasts, suggesting that the inhibition HSP90 $\alpha$ was crucial in the differentiation and formation of osteoclasts. Nevertheless, the inhibition of HSP90 $\alpha$ had no significant effect on PPAR $\gamma$ expression, suggesting a synergistic relationship between HSP90 $\alpha$ and PPAR $\gamma$. These data were consistent with Kim et al (17), who revealed that ligand of PPAR $\gamma$ did not immediately activate the downstream pathway after binding to PPAR $\gamma$, but instead, it bound to HSP90 $\alpha$ before exerting its biological effect. However, the underlying molecular mechanism remained unclear. Considering that PPAR $\gamma$ has the function of nuclear transcription, its function is based on binding to response elements. In the present study, it was theorized that the binding of HSP90 $\alpha$ to PPAR $\gamma$ may promote its transcription into the nucleus. Thus, the nuclear import of PPAR $\gamma$ was observed with a fluorescence microscope, revealing that nuclear import of PPAR $\gamma$ was increased after THP-1 monocytes were induced, and the nuclear import was decreased after inhibition of HSP90 $\alpha$. This indicated that the inhibition of HSP90 $\alpha$ led to the blocking of osteoclast differentiation, which was achieved by inhibiting the nuclear import of PPAR $\gamma$.

Finally, the effects of HSP90 $\alpha$ inhibition on OP in mice were further verified through in vivo experiments. First, a 

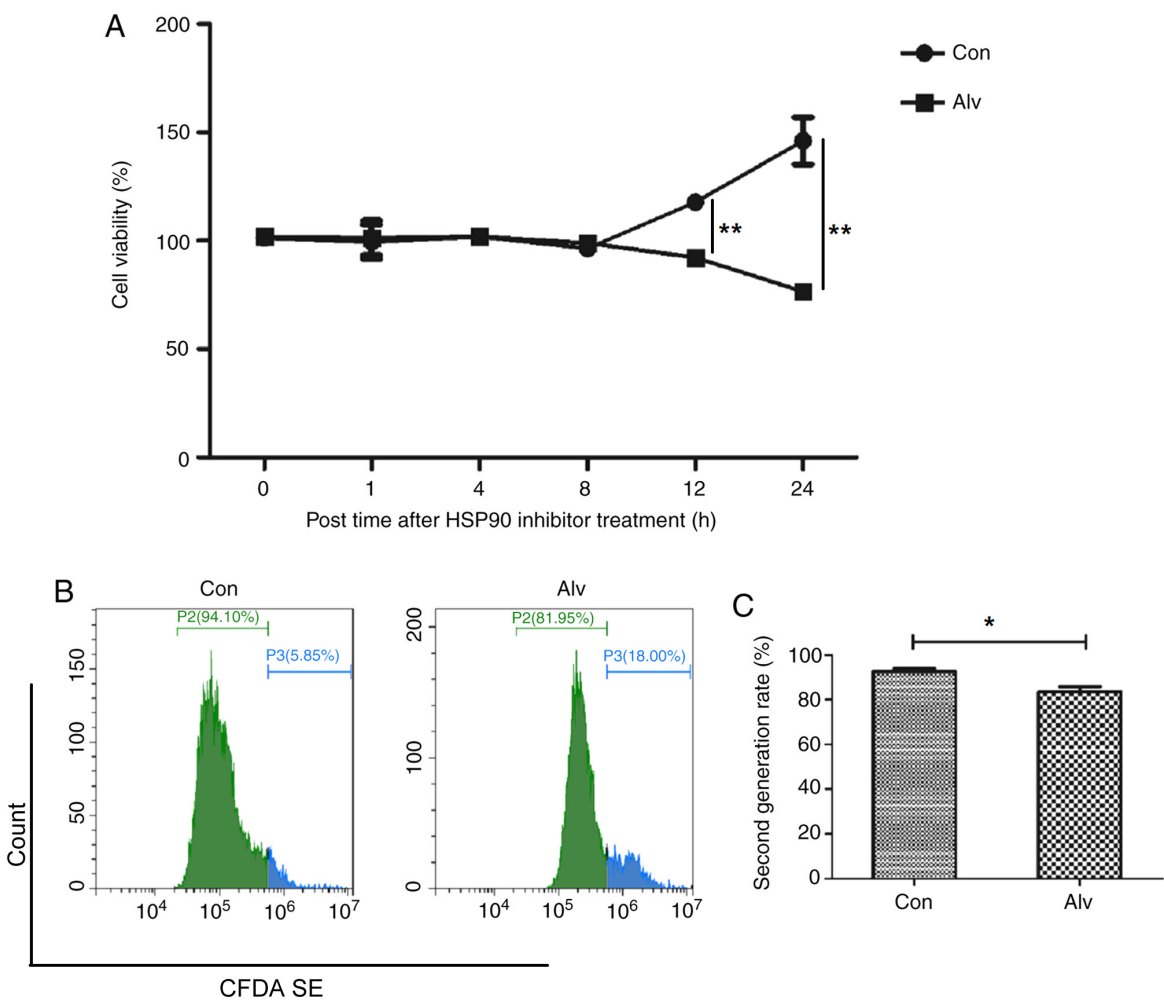

Figure 4. Effect of HSP90 $\alpha$ on the biological proliferative ability of osteoclasts. With treatment of alvespimycin (20 nM), (A) the cell viability of THP-1 cells was determined by CCK-8 assay at designed time-points, and (B and C) proliferation potential was evaluated by CFDA SE-labeled flow cytometric test. ${ }^{*} \mathrm{P}<0.05$ and ${ }^{* *} \mathrm{P}<0.01$. HSP, heat shock protein; Con, control; Alv, alvespimycin.

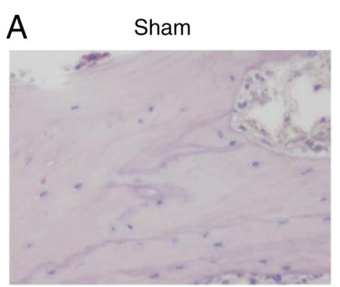

OVX

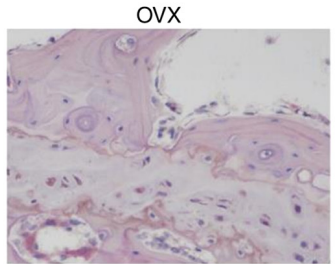

C

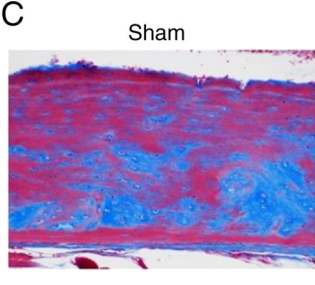

OVX

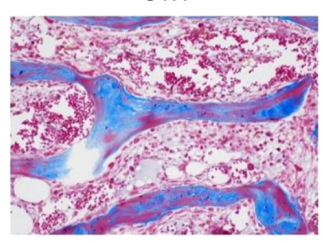

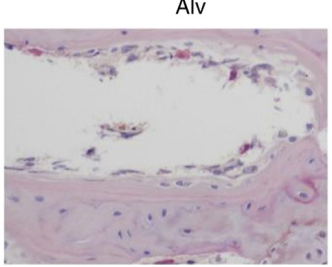

OVX+Alv

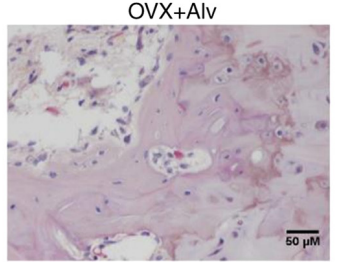

Alv

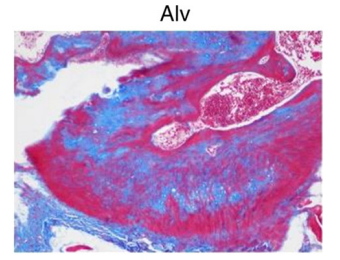

OVX+Alv

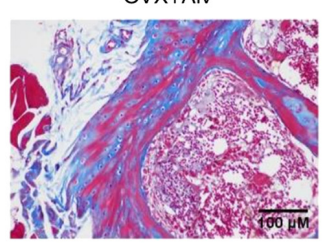

B

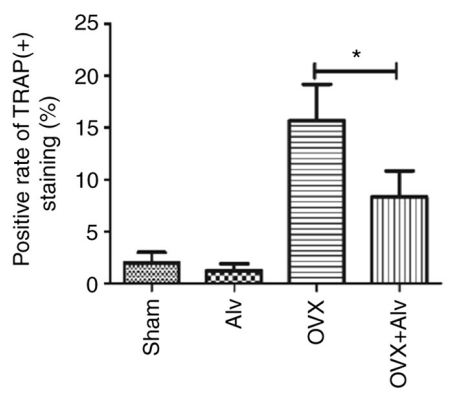

D

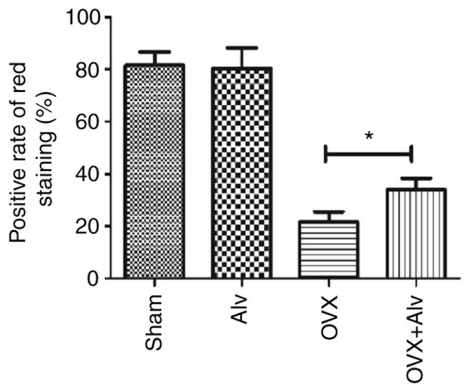

Figure 5. Inhibition of HSP90 $\alpha$ can improve osteoporosis in mice. The osteoporosis mouse model was established by removing ovaries. Then with/without alvespimycin $(25 \mathrm{mg} / \mathrm{kg})$, the degree of osteoporosis was assessed by (A and B) TRAP staining and (C and D) Masson staining. Data are expressed as the mean \pm SD of three separate experiments $(n=5)$. ${ }^{*}<<0.05$. HSP, heat shock protein; Alv, alvespimycin; OVX, ovariectomy. 
mouse model of OP was established by ovariectomy, after which it was used to verify the role of HSP90 $\alpha$. The obtained results revealed that alvespimycin could reduce TRAP staining of bone and relieve OP in mice.

The present study also has a few limitations. Firstly, the specific binding between HSP90 $\alpha$ and PPAR $\gamma$ was not analyzed. Secondly, the effects of other HSPs were not explored. Thus, additional studies are required to confirm these findings.

To sum up, this is the first study, to the best of our knowledge, that reported how inhibition of HSP90 $\alpha$ could block PPAR $\gamma$ and in turn inhibit differentiation in mice osteoclasts. It is considered that inhibition of HSP90 $\alpha$ can block the nuclear import of PPAR $\gamma$, which in turn affects the transcription of corresponding downstream molecules, thereby inhibiting osteoclast differentiation. The aforementioned findings can provide a new direction for the treatment of OP, using HSP90 $\alpha$ as a target and then interfering with the formation of osteoclasts may be the direction of future research.

\section{Acknowledgements}

Not applicable.

\section{Funding}

No funding was received.

\section{Availability of data and materials}

All data generated or analyzed during this study are included in this published article.

\section{Authors' contributions}

JW and YG designed the study. JM, CY and KZ performed the experiments. CW performed the histological examination. XL performed the confocal microscopy analysis. JM, CY and $\mathrm{HZ}$ wrote the paper. $\mathrm{HZ}$ and $\mathrm{CY}$ analyzed and interpreted the data. JW and YG confirm the authenticity of all the raw data. All authors read and approved the final manuscript.

\section{Ethics approval and consent to participate}

All animal experiments were undertaken in accordance with the National Institute of Health 'Guide for the Care and Use of Laboratory Animals' (NIH Publication no. 85-23, National Academy Press, Washington, DC, revised 1996), with the approval of the Laboratory Animal Center and Ethics Committee of Changzheng Hospital (approval ID: \#CZ2019112130).

\section{Patient consent for publication}

Not applicable.

\section{Competing interests}

The authors declare that they have no competing interests.

\section{References}

1. Shanb AA and Youssef EF: The impact of adding weight-bearing exercise versus nonweight bearing programs to the medical treatment of elderly patients with osteoporosis. J Family Community Med 21: 176-181, 2014.

2. Lin J, Zhu J, Wang Y, Zhang N, Gober HJ, Qiu X, Li D and Wang L: Chinese single herbs and active ingredients for postmenopausal osteoporosis: From preclinical evidence to action mechanism. Biosci Trends 11: 496-506, 2017.

3. Hamdy NA: Targeting the RANK/RANKL/OPG signaling pathway: A novel approach in the management of osteoporosis. Curr Opin Investig Drugs 8: 299-303, 2007.

4. Li X, Jie Q, Zhang H, Zhao Y, Lin Y, Du J, Shi J, Wang L, Guo K, Li Y, et al: Disturbed MEK/ERK signaling increases osteoclast activity via the Hedgehog-Gli pathway in postmenopausal osteoporosis. Prog Biophys Mol Biol 122: 101-111, 2016.

5. Granéli C, Karlsson C, Brisby H, Lindahl A and Thomsen P: The effects of PPAR- $\gamma$ inhibition on gene expression and the progression of induced osteogenic differentiation of human mesenchymal stem cells. Connect Tissue Res 55: 262-274, 2014.

6. Wang J, Wang G, Gong L, Sun G, Shi B, Bao H and Duan Y: Isopsoralen regulates PPAR- $\gamma / \mathrm{WNT}$ to inhibit oxidative stress in osteoporosis. Mol Med Rep 17: 1125-1131, 2018.

7. Wan Y, Chong LW and Evans RM: PPAR-gamma regulates osteoclastogenesis in mice. Nat Med 13: 1496-1503, 2007.

8. Rossi A, Kapahi P, Natoli G, Takahashi T, Chen Y, Karin M and Santoro MG: Anti-inflammatory cyclopentenone prostaglandins are direct inhibitors of IkappaB kinase. Nature 403: 103-108, 2000.

9. Zhang M, Qian C, Zheng ZG, Qian F, Wang Y, Thu PM, Zhang X, Zhou Y, Tu L, Liu Q, et al: Jujuboside A promotes A $\beta$ clearance and ameliorates cognitive deficiency in Alzheimer's disease through activating Axl/HSP90/PPAR $\gamma$ pathway. Theranostics 8: 4262-4278, 2018

10. Hu Y, Bobb D, He J, Hill DA and Dome JS: The HSP90 inhibitor alvespimycin enhances the potency of telomerase inhibition by imetelstat in human osteosarcoma. Cancer Biol Ther 16: 949-957, 2015.

11. Mellatyar H, Talaei S, Pilehvar-Soltanahmadi Y, Barzegar A, Akbarzadeh A, Shahabi A, Barekati-Mowahed $M$ and Zarghami N: Targeted cancer therapy through 17-DMAG as an Hsp90 inhibitor: Overview and current state of the art. Biomed Pharmacother 102: 608-617, 2018.

12. Tsai YC, Leu SY, Chen SY, Kung CW, Lee YM, Liu YP, Yen MH and Cheng PY: 17-DMAG, an Hsp90 inhibitor, ameliorates ovariectomy-induced obesity in rats. Life Sci 232: 116672, 2019.

13. Hertlein E, Wagner AJ, Jones J, Lin TS, Maddocks KJ, Towns WH III, Goettl VM, Zhang X, Jarjoura D, Raymond CA, et al: 17-DMAG targets the nuclear factor-kappaB family of proteins to induce apoptosis in chronic lymphocytic leukemia: Clinical implications of HSP90 inhibition. Blood 116: 45-53, 2010.

14. Ge J, Normant E, Porter JR, Ali JA, Dembski MS, Gao Y, Georges AT, Grenier L, Pak RH, Patterson J, et al: Design, synthesis, and biological evaluation of hydroquinone derivatives of 17-amino-17-demethoxygeldanamycin as potent, water-soluble inhibitors of Hsp90. J Med Chem 49: 4606-4615, 2006.

15. Wu JS, Tsai HD, Cheung WM, Hsu CY and Lin TN: PPAR- $\gamma$ ameliorates neuronal apoptosis and ischemic brain injury via suppressing NF- $\kappa \mathrm{B}$-driven $\mathrm{p} 22$ phox transcription. Mol Neurobiol 53: 3626-3645, 2016.

16. Zininga T, Ramatsui $\mathrm{L}$ and Shonhai A: Heat shock proteins as immunomodulants. Molecules 23: 2846, 2018.

17. Kim HJ, Hwang NR and Lee KJ: Heat shock responses for understanding diseases of protein denaturation. Mol Cells 23: 123-131, 2007.

18. Nguyen MT, Csermely P and Sóti C: Hsp90 chaperones PPAR $\gamma$ and regulates differentiation and survival of 3T3-L1 adipocytes. Cell Death Differ 20: 1654-1663, 2013.

19. Lee KJ, Ha ES, Kim MK, Lee SH, Suh JS, Lee SH, Park KH, Park JH, Kim DJ, Kang D, et al: CD36 signaling inhibits the translation of heat shock protein 70 induced by oxidized low density lipoprotein through activation of peroxisome proliferators-activated receptor gamma. Exp Mol Med 40: 658-668, 2008.

20. Chen TH, Shih CY, Hsu WL and Chou TC: Mechanisms of nifedipine-downregulated CD40L/sCD40L signaling in collagen stimulated human platelets. PLoS One 10: 0127054, 2015. 